\title{
The Impact of Using Computed Tomography And Positron Emission Tomography Image Fusion in Radiotherapy of Lung Cancer
}

\author{
AE Amin ${ }^{1}$, MS Hassan Talaat ${ }^{2 *}$, SM EISayed ${ }^{1}$ and AM Hamed ${ }^{1}$ \\ ${ }^{1}$ Ain shams oncology center, Ain-shams University, Egypt. \\ ${ }^{2}$ Physics Department, Ain-shams University, Egypt
}

Submission: May 01, 2017; Published: May 24, 2017

*Correspondence Address: MS Hassan Talaat, Physics Department, Faculty of Science, Ain-shams University, Cairo, Egypt

\begin{abstract}
Purpose: To validate a computed tomography (CT) and Positron Emission Tomography (PET) image fusion procedure and to evaluate its usefulness to facilitate tumor definition and physical assessment of treatment planning using dose volume histogram (DVH) in threedimensional conformal radiation therapy (3D-CRT) for lung cancer.
\end{abstract}

Methods and Materials: Eleven patients were assessed by CT and PET-CT fusion images before radiotherapy. The patients were placed in the same position during CT and PET using TF TOF Philips 64 slices PET-CT machine. Matching was achieved by using XIO planning system software. 22 planning target volume (PTV's) and 22 plans were performed for all patients; 2 PTVs and 2 plans for every patient. First PTV and plan were generated using CT images called PTVCT and CT plan respectively, the other PTV and plan generated using PET images called PTVPET and PET plan respectively. V20 of the lung, Esophagus mean dose, heart mean dose, conformity index (CI) and homogeneity index (HI) were calculated and compared for every patient.

Results: Fusion of anatomic and metabolic data decreased the target volume definition by exclusion of lung atelectasis or consolidation in 7 patients otherwise fusion increased the target volume by corrects staging of lymph nodes (LN) for 4 patients. In these 7 patients, DVH revealed that the lung volume irradiated at $20 \mathrm{~Gy}\left(\mathrm{~V}_{20}\right)$ was decreased; tumor volume irradiated at the $95 \%$ isodose $\left(\mathrm{V}_{95}\right)$ was optimized. In other 4 patients $V_{20}$ of the lung was increased due to target volume increased. Esophagus mean dose and heart mean dose changed according to how much target volume extended near to them. CI an HI calculated for PTVPET and exhibits very optimal results in compare with CT images.

Conclusion: We have validated CT and PET lung image fusion for accurate detection of lung cancer volumes, which improved the radiation treatment procedure of the lung.

Keywords: Computed Tomography (CT); Conformity index (CI); Homogeneity index (HI); Positron Emission Tomography (PET); Planning Target Volume of Computed Tomography (PTVCT); Planning Target Volume of Positron Emission Tomography (PTVPET) and Three dimensional conformal radiation therapy (3DCRT)

\section{Introduction}

3DCRT is used in many radiotherapy departments for a variety of tumor sites. Because it irradiates tumors while simultaneously sparing organs at risk (OARs) adjacent to the planning target volume (PTV) [1]. Using PET-CT image fusion rather than conventional CT images in the treatment planning of lung cancer, the treatment planning system (TPS) has ability to make fusion between CT images and PET images which help us in target volume delineation as well as all (OARs). In patients with lung cancer, considered for radiation treatment, accurate assessment of hilar, mediastinal lymph node (LN) and atelectasis or consolidation involvement is of utmost importance.

The extent of the tumor will not only influence the treatment intention, i.e. curative or palliative, but also the volumes to be treated, therefore the dose to be expected. Indeed, several authors have suggested that a dose-volume effect for radio pneumonitis exists [2-5]. Different parameters, derived from 
(DVH's), have been correlated with the incidence of pneumonitis observed, e.g. $V_{20}$ of the lung [2], and other complication of different OAR dose such as esophagus mean dose and mean heart dose. In recent years, PET-CT has been shown by several authors to improve the accuracy of mediastinal LN staging and exclusion of consolidation compared to CT alone [7-10]. thoracic lymph nodes (LNs) on fluoro-2-deoxy-D-glucose positron emission tomography (FDG-PET). In non-small cell lung cancer (NSCLC) patients referred for loco regional LN staging, this study prospectively examined to what extent localization of LNs at PET reading could be improved by visual correlation with computed tomography (CT), or by PET+CT fusion images. Fifty-six patients with potentially operable NSCLC underwent CT, PET and surgical staging. Prospective reading was performed for CT, PET without CT, PET-CT visual correlation (not fusion) and PET+CT fusion. Reading was blinded to surgical pathology data and note on a standard LN map. In the evaluation per individual LN station, CT was accurate in $87 \%$, PET in 91\% and visual correlation and fusion in $93 \%$. They conclude that in the exact localization of metastatic thoracic LNs, the accuracy of reading of PET is increased if the PET images can be visually correlated with CT images.

In a study of Erdi et al. [12] many patients with non-small cell lung cancer (NSCLC); Imaging with fluoro-2-deoxyglucose (FDG) PET in conjunction with CT, can improve the accuracy of lesion definition. In their study, they investigated the potential benefits of incorporating PET data into the conventional treatment planning of NSCLC. Case-by-case, they prospectively analyzed planning target volume (PTV) and lung toxicity. The

Materials and Methods

Table 1: Dose constrains of target and OAR of 3DCRT lung case.
PTV, along with the gross target volume (GTV) and all organs at risk, was first delineated using the CT data set. (DVHs) for lesion and normal organs were generated using both CT-based and PET/CT-based treatment plans. In seven out of 11 cases, they found an increase in PTV volume (average increase of 19\%) to incorporate distant nodal disease. between these patients; the highest normal tissue complication probability (NTCP) for lung was $22 \%$ with combined PET/CT plan and $21 \%$ with CT plan. In other four patients PTV was decreased an average of $18 \%$. The reduction of PTV in two of these patients was due to excluding atelectasis and removing the target volume to avoid delivering radiation doses to nearby spinal cord or heart. Jeffrey et al. [13] had performed a study to determine the impact of FDG-PET and $\mathrm{CT}$ on radiation therapy. Both the $\mathrm{CT}$ and fused PET/CT image data sets were transferred to the radiation treatment planning system for contouring. Each FDG-PET study was reviewed with the interpreting nuclear radiologist before contouring of target volume. The fused PET/CT images were used to develop the three-dimensional conformal radiation therapy (3DCRT) plan. Other physician, blinded to the results of PET, contoured the gross tumor volumes (GTV) and planning target volumes (PTV) from the CT images. The two 3DCRT plans for every patient were compared with respect to the GTV, PTV, mean lung dose, volume of health lung receiving $>20 \mathrm{~Gy}\left(\mathrm{~V}_{20}\right)$, and mean esophageal dose. They conclude that increase in the target volumes led to increase in the mean lung dose, $\mathrm{V}_{20}$, and mean dose of esophageal. Decreases in the target volumes in the patients with atelectasis led to decreases in these normal-tissue toxicity parameters.

\begin{tabular}{|c|c|c|c|c|c|}
\hline Structure & $\begin{array}{c}\text { Prescribed dose } \\
\text { (cGy) }\end{array}$ & Mean dose (cGy) & Under dose (cGy) & Max. dose (cGy) & $\begin{array}{c}\text { Volume received } \\
\text { (20 Gy) }\end{array}$ \\
\hline PTV & 6000 & & 5700 & 6420 \\
\hline Heart & & 2600 & & & \\
\hline Esophagus & & 3400 & & & $\begin{array}{c}\text { Less than 30\% of } \\
\text { volume }\end{array}$ \\
\hline Both Lungs & & & & & \\
\hline
\end{tabular}

In this study we include eleventh lung cancer patients. We selected patients from the treatment list of AIN SHAMS oncology center 2 year ago. In this study, patients who had lung cancer were divided into two groups according to their size of PTV in both CT images and PET images. Patients were distributed among these two groups according to the PTV-CT volume in cubic centimeter. One group has PTV-CT larger than PTV-PET in volume and represents $63.6 \%$ of all cases, the other has PTVCT smaller than PTV-PET in volume and represents $36.4 \%$ of all cases. PET and CT images had $5 \mathrm{~mm}$ spacing over the entire treatment area "chest".
Only two treatment volumes PTVCT, PTVPET and other OARs were defined, the PTV included ( (GTV + CTV) " $1 \mathrm{~cm}$ margin from GTV" + margin (1.5 cm whole around CTV for (PTV)). In this study, the OARs are Esophagus, Heart, both lungs minus PTV and Spinal cord, and we will perform two plans one plan focus with PTVCT as a target and called CT plan and the other plan focus with PTVPET and called PET plan. XIO® planning System (Elekta medical systems) was used in this study to perform the 3DCRT and in calculation process depends on the measurements of physical \& geometrical data of ELEKTA Linear accelerator for both $6 \& 15 \mathrm{MV}$ photon energies. We are aiming in this study to 


\section{Cancer Therapy \& Oncology International Journal}

detect the effect of change the target volume by trying 2 target volumes with the same plan. We used only the dose constrains of the QUANTIC [14]. The prescribed dose in this treatment course was 60 Gy by $2 \mathrm{~Gy} /$ fraction, 6 weeks. Constrains of the treatment planning are shown in (Table 1) [6].

The dose volume histograms (DVHs) of both PTV and all OARs were calculated. DVH of the PTV, the D95 (the minimum absorbed dose that covers $95 \%$ of the volume of the PTV) was estimated. For Both Esophagus and Heart The mean dose were estimated. For both lungs volumes which receive less than $20 \mathrm{~Gy}$ was estimated. 'The concept of HI was developed as an extension of section-by section dosimetric proposed guidelines for routine evaluation of stereotactic radiotherapy (SRT) plans based on several parameters and HI was described as,

$$
\mathrm{HI}=\left(\mathrm{D}_{2 \%}-\mathrm{D}_{98 \%}\right) / \mathrm{D}_{50 \%}
$$

Where D2\% represents the dose to 2\% of PTV, D98\% represents the dose to $98 \%$ of PTV and D50\%represent the dose to $50 \%$ of PTV. The conformity index (CI) is defined as the ratio of the total volume receiving at least the prescription dose "Vp", to the target volume receiving at least the prescription dose "Vtp"CI=Vp/Vtp..

Table 2: The physical parameters with the different treatment plans.

\begin{tabular}{|c|c|c|}
\hline \multirow{2}{*}{ Case no. } & \multicolumn{2}{|c|}{ The Target volumes } \\
\hline & PTV-CT & PTV-PET \\
\hline 1 & 403.29 & 363.5 \\
\hline 2 & 392.23 & 323.23 \\
\hline 3 & 465.34 & 330.47 \\
\hline 4 & 549.83 & 412.67 \\
\hline 5 & 605.1 & 375.77 \\
\hline 6 & 765.95 & 454.39 \\
\hline 7 & 969.82 & 643.78 \\
\hline 8 & 1370.95 & 742.66 \\
\hline 9 & 878.8 & 685.13 \\
\hline 10 & 369.16 & 531.22 \\
\hline \multirow[t]{2}{*}{11} & 373.71 & 515.16 \\
\hline & PTV-CT & PTV-PET \\
\hline 1 & 403.29 & 363.5 \\
\hline 2 & 392.23 & 323.23 \\
\hline 3 & 465.34 & 330.47 \\
\hline 4 & 549.83 & 412.67 \\
\hline 5 & 605.1 & 375.77 \\
\hline 6 & 765.95 & 454.39 \\
\hline 7 & 969.82 & 643.78 \\
\hline 8 & 1370.95 & 742.66 \\
\hline 9 & 878.8 & 685.13 \\
\hline 10 & 369.16 & 531.22 \\
\hline 11 & 373.71 & 515.16 \\
\hline
\end{tabular}




\section{Cancer Therapy \& Oncology International Journal}

These dose values were used in the comparison of the two CT and PET plan. For each case, we perform 2 plans; Table 2 shows the size of target volume of these plans. Patients from case number 1 to case number 7 have large PTVCT than PTVPET, while patients from number 7 to number 11 have small PTVCT than PTVPET..

\section{Results}

In this study, each case has two plans, Both PTVCT and PTVPET are covered with $95 \%$ in both CT and PET plans regardless the target size. In conformal radiotherapy planning using CT plan dose distribution covered PTVCT; the un-coverage of PTVPET represents a serious issue in radiotherapy planning as we pointed out the PTVCT is fully covered by 95\%, while PTVPET is not covered by that level. The un-coverage of PTVPET may lead to treatment failure Figure 1.

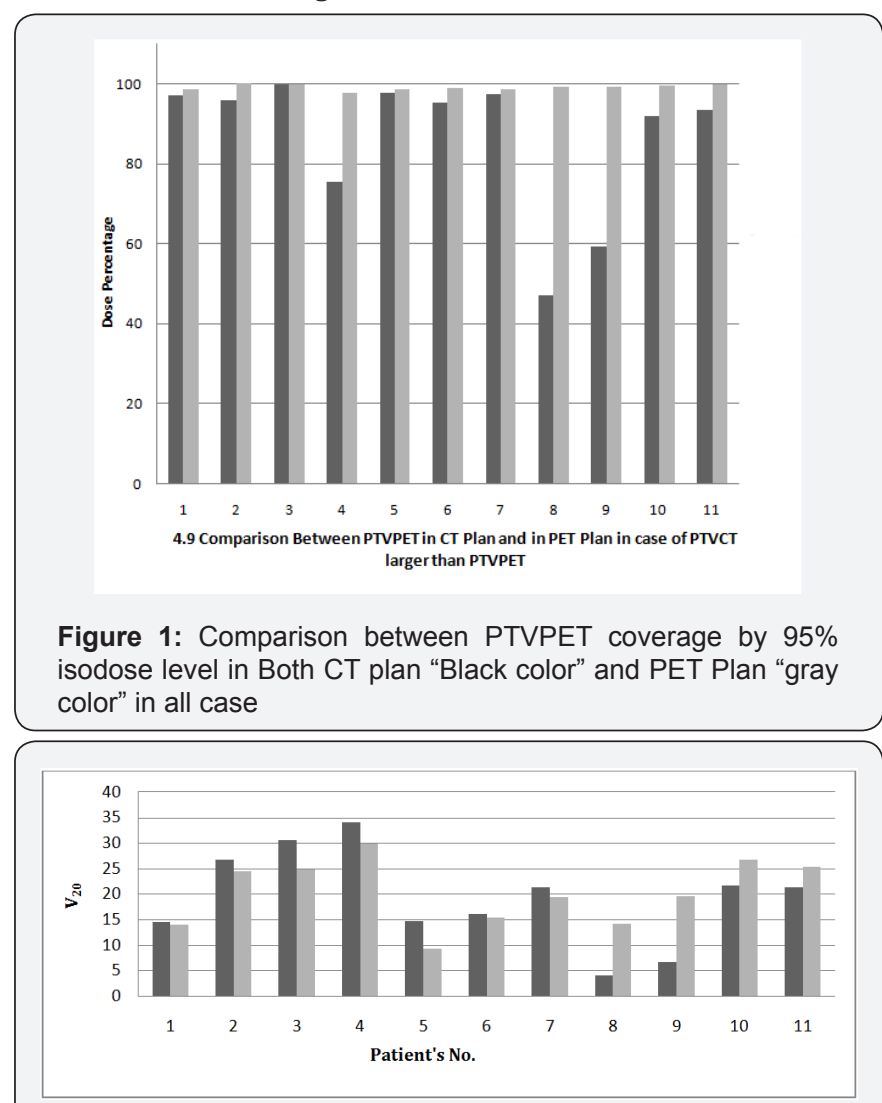

Figure 2: Comparison between Lung PET in Both CT plan "Black color" and PET Plan "gray color" in all cases.

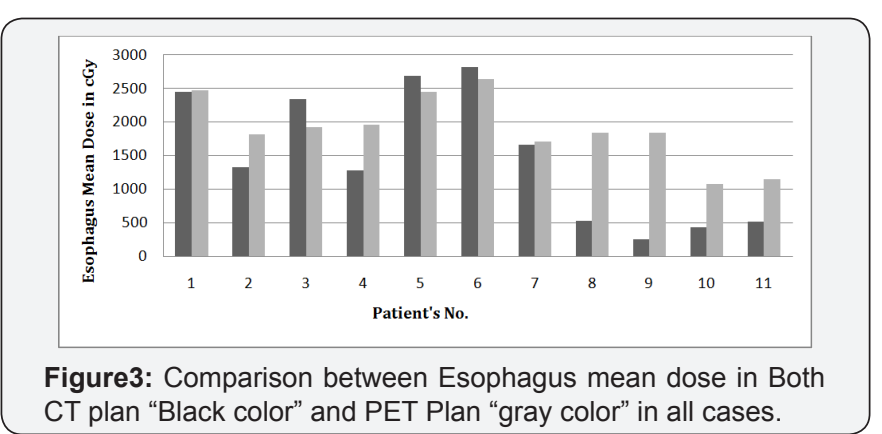

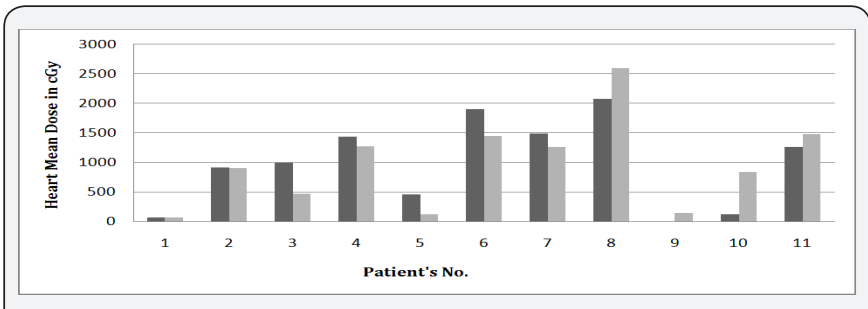

Figure 4: Comparison between Heart mean dose in Both CT plan "Black color" and PET Plan "gray color" in all cases.

Dose volume histograms (DVH's) were calculated and generated based on 3D reconstructed images for PTV and all OARs in all treatment plans. All plans fulfilled dose volume constrains and verified by using DVHs according to "ICRU 83". In all PET plan, the auto contoured Lung minus PTVPET defines as (Lung PET) fulfilled constraint and the volume of lung PET received 20 Gy is less than $30 \%$ of total Lung PET volume (QUANTIC, 2010) [14] in all PET plan Figure 2 Show the lung V20 for all 11 cases, each case have 2 plans one "black columns" defined lung dose on CT plan and other "gray color" defined lung dose in PET plans. We notice that is the first seven cases opposite in comparison to last four cases in lung PET dose through both CT plan and PET plan. The first seven cases have PTVCT larger than PTVPET in volume therefore; CT plan will have been larger irradiation area then, lung PET in CT plan "black columns" will be higher than lung PET in PET plan "gray columns".

On the other hand, the last four cases have PTVCT smaller than PTVPET then, smaller irradiation area so, and lung PET in PET plan will be larger than lung PET in CT plan. The applied dose volume constrains of esophagus according to (QUANTIC, 2010) [14] were: The mean dose $\leq 30$ Gy. Figure 3 shows the comparison between esophagus mean dose in CT plan "Black columns" and in PET plan "gray columns" for 11 patients. In this work we notice that dose to esophagus may be increased CT plan "black columns" more than that in PET plan "gray columns", The reason of that is the dependency of the mass location in relation to esophagus. If the PTV is in the peripheral side of the lung will be lower than that in cases with PTV in the middle of the lung. Usually PET plan using PET images changes the target volume to include hilar or medistineal positive lymph node (i.e. size of target volume increased), this cause the large mass of target is to be close to the esophagus, and accordingly increases of its dose.

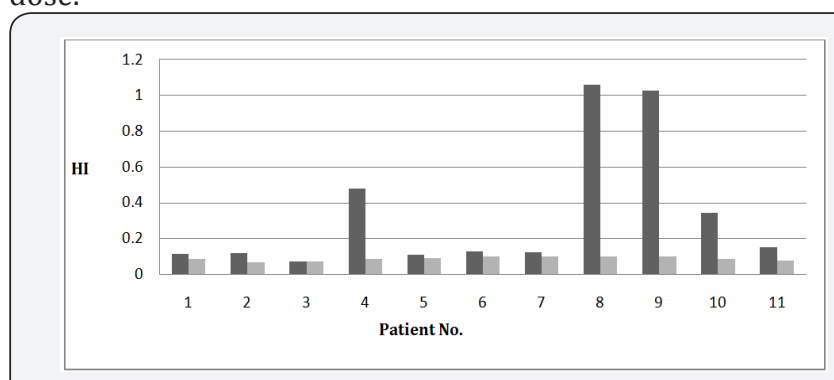

Figure 5: Comparison between Cl of PTVPET in Both CT plan "Black color" and PET Plan "gray color" in all cases. 


\section{Cancer Therapy \& Oncology International Journal}

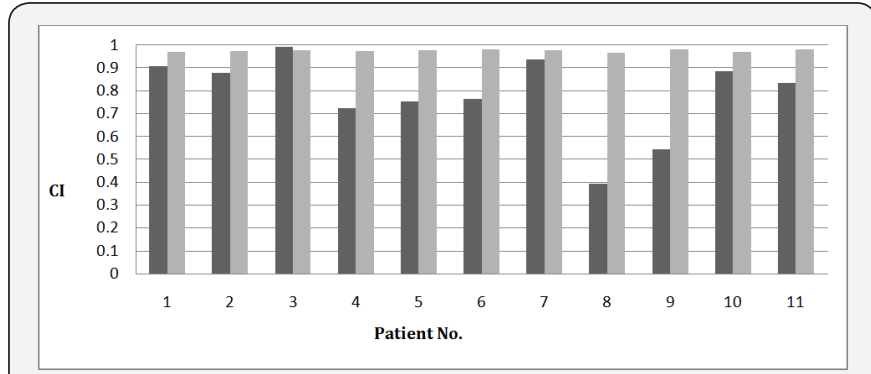

Figure 6: Comparison between Cl of PTVPET in Both CT plan "Black color" and PET Plan "gray color" in all cases.

On the other hand, the esophagus dose may be decreases by doing CT plan more than PET plan, due to the position of the PTV, which is far from esophagus. Figure 2 shows the comparison between the CT plan "Black color" and PET plan "gray color" regarding to esophagus mean dose for 11 patients. Heart dose volume constrains applied in this study are: Mean dose of heart $\leq 26$ Gy (QUANTIC, 2010) [14]. Figure 4 shows a comparison between heart in CT plans and PET plans, which have been done for 11 patients. In the first 6 cases the mean heart dose in PTVCT plans "black columns" is higher than that in PTVPET "gray columns" and we got exactly the opposite in the other 5 cases. Now we notice that is when, PTVCT is larger than PTVPET the heart dose in CT plan "black columns" will be higher than in PET plan "gray columns" and vice versa. This is due to the increases of irradiation area and the position of PTV regarding to the heart.

Figure 4 shows PTVPET in PET plans "gray columns" has HI values less than PTVCT in PET plan "black columns". CI generally used to indicate the portion of prescription dose that delivered inside the PTV. CI of one indicates that $100 \%$ of prescribed dose delivered to the PTV and no dose delivered to adjacent tissue, CI value is always close to value of one in most clinical cases. Lower CI values indicate poorer dose conformity to the PTV. Figure 5 shows PTVPET in PET plans "gray columns" have HI low values "near to zero" than PTVCT in PET plan "black columns" which is optimal results for PTVPET "metabolic target volume". Fig (6) CI generally used to indicate the portion of prescription dose that delivered inside the PTV. CI of one indicates that $100 \%$ of prescribed dose delivered to the PTV and no higher dose delivered to adjacent tissue, CI value is always close to value of one in most clinical cases. Lower CI values indicate poorer dose conformity to the PTV. Figure 6 represents CI of PTVPET in both CT plan "black columns" and in PET plan "gray columns". we can notice that in all plans, the CI values of PTVPET in PET plan "gray color" is almost value of one and this means that PTVPET in PET plan have good conformity. On the other hand, in all plans CI values of PTVPET in CT plan have a lower values so, PTVPET in CT plan have poor conformity than PTVPET in PET plan, which is accepted because of PTVPET "metabolic target volume" is fully covered target.

\section{Discussion}

The use of FDG-PET examinations in the assessment of lung cancer is increasingly included in new research. Various studies have addressed the value of PET in change the target volume. Little is known about PET and its accuracy with respect to size and shape of the primary tumor. The literature available hints that the size may be depicted quite accurately compared to CT images. However, due to the increased uptake of FDG the size of the tumor itself may be rather over than underestimated. For lung cancer, Erdi et al. [12] and Jeffrey et al. [13] found the PTV, along with PTV and all OARs, was first delineated using the CT data set, DVHs for tumor and normal organs were generated using both CT-based and PET-CT based treatment plans. In seven out of 11 cases, they found an increase in PTV volume (average increase of $19 \%$ ) to incorporate distant nodal disease. between these patients; the highest normal tissue complication probability (NTCP) for lung was $22 \%$ with combined PET/CT plan and $21 \%$ with CT plan. In other four patients PTV was decreased an average of $18 \%$ and these results agree with our results in changing in target volume. Therefore, we decided to change the concept of including the atelectasis or consolidation, lymph nodes. However, following this concept, the information given by PET still added considerably to the information provided by CT, leading to substantial changes, mainly the reduction of the size of the treatment volume and also to increase the target volume.

In the group of patients with consolidation or atelectasis, the contribution of the PET scan led to a significantly higher rate of field sizes changes compared to the other cases. Mostly, the field sizes were reduced because PET images excluded non-metabolic active cells leading to reduce the target volume. This finding may be explained by the fact that the margins between tumor and atelectasis cannot be drawn clearly using the morphological information provided by CT alone. Due to this uncertainty, larger PTV's may be defined including larger volumes of healthy lung tissue, esophagus tissue and heart.

PET helps patients have positive lymph nodes, the target volume were increased due to inclusion of them; thus organs at risk dose increases but with high tumor control than in CT planning. It is reasonable to assume that the impact of PET in 3DCRT would be even greater, considering the size of the target volume in itself. The impact of PET might also be greater, if, instead of mere visual comparison, image fusion were used for matching PET and CT data. While we are currently working on these aspects, the results obtained until now show clearly that in using PET in the 3DCRT of lung cancer, a substantial reduction in the amount of healthy lung irradiated can be expected, especially in.

\section{Conclusion}

This study investigated the integration of CT and PET image data during curative 3DCRT for patients with lung cancer. The functional PET studies appear to complement CT information 


\section{Cancer Therapy \& Oncology International Journal}

and hopefully will result in improved treatment plans by changing the tumor volume sometimes to exclude atelectasis or consolidation and other to include any positive lymph node and improve target coverage, $\mathrm{CI}$ and $\mathrm{HI}$ and keep all organs at risk away from any toxicity or complication.

\section{References}

1. Klaus B, Matthias G, Gerd M (2007) A comparison between 2-Step IMRT and conventional IMRT planning. Radiotherapy and Oncology 84(3): 298-306.

2. Graham MV, Purdy JA, Emami B (1995) Preliminary results of a prospective trial using three dimensional radiotherapy for lung Cancer Int J Rad Oncol Biol Phys 33: 993-1000.

3. Marks LB, Munley MT, Bentel GC (1997) Physical and biological predictors of changes in whole-lung function following thoracic irradiation. Int J Rad Oncol Biol Phys 39: 563-570.

4. Martel MK, Ten Haken RK, Hazuka M (1994) B. Dose-volume histogram and 3-D treatment planning evaluation of patients with pneumonitis. Int J Rad Oncol Biol Phys 28: 575-581.

5. Patz EF, Lowe VJ, Goodman PC, Herndon J (1995) Thoracic nodal staging with PET imaging with 18FDG in patients with bronchogenic carcinoma. Chest 108: 1617-1621.

6. Nestle U, Walter K, Schmidt S, Sybrecht GW, Schnabel K, et al. (1999) 18F-deoxyglucose positron emission tomography (FDG PET) for the planning of radiotherapy in lung cancer: High impact in patients with atelectasis. Int J Radiat Oncol Biol Phys 44: 593-59.

7. Sasaki M, lchiya Y, Kuwabara Y, et al. (1996) The usefulness of FDG positron emission tomography for the detection of mediastinal lymph node metastases in patients with non-small cell lung cancer: a L.J.

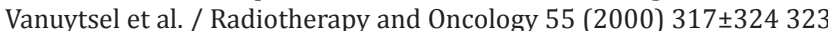
comparative study with X-ray computed tomography. Eur J Nucl Med 23: 741-747.

8. Valk PE, Pounds TR, Hopkins DM, et al. (1995) Staging non-small cell lung cancer by whole-body positron emission tomographic imaging. Ann Thorac Surg 60(6): 1573-1582.

9. Wahl RL, Quint LE, Greenough RL, White RI, Orringer MB, et al. (1994) Staging of mediastinal non-small cell lung cancer with FDG PET. CT and fusion images: Preliminary prospective evaluation. Radiology 191: 371-377.

10. Chin R, Ward R, Keyes JW (1995) Mediastinal staging of non-small cell lung cancer with positron emission tomography. Am J Respir Crit Care Med 152: 2090-2096.

11. Vansteenkiste JF (1998) FDG-PET scan in potentially operable nonsmall cell lung cancer: do anatometabolic PET-CT fusion images improve the localization of regional lymph node metastases. Eur J Nucl Med 25(11): 1495-501.

12. Erdi YE, Rosenzweig K, Erdi AK, Macapinlac HA, Hu YC, et al (Radiotherapy Treatment Planning for patients with non-small cell lung cancer using positron emission tomography (PET). Radiother Oncol 62: 51-60.

13. Jeffrey Bradley, Dehdashti F, Siegel BA, Bosch W, Bertrand RJ, et al. (2004) Impact of FDG-PET on radiation therapy volume delineation in non-small-cell lung cancer. Int J Radiation Oncology Biol Phys 59(1): 78-86.

14. Lawrence, Yorke, Andrew, Randall K Ten Haken, Avraham Eisbruch, et al. (2010) Use of Normal Tissue Complication Probability Models in the Clinic. Int J Radiation Oncology Biol Phys 76(3): S10-S19.

\section{Your next submission with Juniper Publishers will reach you the below assets}

- Quality Editorial service

- Swift Peer Review

- Reprints availability

- E-prints Service

- Manuscript Podcast for convenient understanding

- Global attainment for your research

- Manuscript accessibility in different formats

( Pdf, E-pub, Full Text, Audio)

- Unceasing customer service

Track the below URL for one-step submission https://juniperpublishers.com/online-submission.php 